\title{
Reflets
}

Revue d'intervention sociale et communautaire

\section{L’engagement militant en service social}

\section{Isabelle Côté, David Buetti, Simon Lapierre et Patrick Ladouceur}

Volume 23, numéro 2, automne 2017

URI : https://id.erudit.org/iderudit/1043300ar

DOI : https://doi.org/10.7202/1043300ar

Aller au sommaire du numéro

Éditeur(s)

Reflets, Revue d'intervention sociale et communautaire

ISSN

1203-4576 (imprimé)

1712-8498 (numérique)

Découvrir la revue

Citer ce document

Côté, I., Buetti, D., Lapierre, S. \& Ladouceur, P. (2017). L'engagement militant en service social. Reflets, 23(2), 10-14. https://doi.org/10.7202/1043300ar d'utilisation que vous pouvez consulter en ligne.

https://apropos.erudit.org/fr/usagers/politique-dutilisation/ 


\title{
L'engagement militant en service social
}

\author{
Isabelle Côté, Ph. D. \\ Professeure adjointe \\ École de service social, Université Laurentienne
}

David Buetti, MSS

Candidat au doctorat

École interdisciplinaire des sciences de la santé, Université d'Ottawa

Simon Lapierre, $\mathrm{Ph} . \mathrm{D}$.

Professeur agrégé

École de service social, Université d'Ottawa

Patrick Ladouceur, MSS

Candidat au doctorat

École de service social, Université d'Ottawa

Si plusieurs écrits ont permis de mieux rendre compte des motifs qui poussent des individus et des groupes à se joindre à différents mouvements sociaux (voir Mathieu, 2004), l'apport des travailleuses et travailleurs sociaux au sein de ces mouvements est souvent passé sous silence. Pourtant, le service social structurel est né par le biais du militantisme (voir l'éditorial du premier numéro du présent volume : Lapierre, et collab., 2017). Comme il est mentionné dans le numéro précédent, l'histoire montre que les travailleuses et travailleurs sociaux ont contribué activement aux luttes pour le changement social, tant par leurs pratiques sociales novatrices que par leurs réflexions intellectuelles visant une société plus juste et inclusive (Bent-Goodley, 2015).

Cette tradition militante en service social peut être liée au désir des intervenantes et intervenants de mettre en place des moyens concrets visant la justice sociale en société (Bent-Goodley, 2015). Dans cette optique, nous avançons que le service social constitue un terreau fertile pour l'action sociale et militante, en raison, entre autres, des valeurs portées par la profession. En effet, au sein d'une discipline qui favorise une réflexion continue permettant de lier les situations vécues individuellement aux structures sociales sous-jacentes (Deslauriers et Turcotte, 2015; Molgat, 2015), l'importance du changement social se veut indéniable. 
Le militantisme se veut pluraliste et fait référence à une gamme d'actions et de stratégies de nature politique permettant de lutter collectivement et solidairement contre les injustices créées par les groupes dominants (Fillieule, Agrikoliansky et Sommier, 2010). Une panoplie de causes sociales sont défendues par les militantes et militants, par différents moyens tels que des mobilisations citoyennes, des regroupements politiques, des manifestations et des pétitions en ligne, des réseaux sociaux et des blogues, et des recherches militantes et critiques (Russell, 2015). Pensons également aux intervenantes et intervenants qui œuvrent au sein de milieux qui favorisent les principes de l'action communautaire dans le cadre de leurs actions et de leurs pratiques (Lavoie et PanetRaymond, 2014). Dépassant une logique de services, les actions et les pratiques qui se fondent sur les principes de l'action communautaire tendent non seulement à répondre aux besoins d'une population précise, mais aussi à favoriser le changement social à long terme en ciblant les causes structurelles des injustices qu'ont vécues les individus et groupes avec qui les intervenantes et intervenants s'associent (Hanley, Kruzynski et Shragge, 2013).

Par ailleurs, si des intervenantes et intervenants sociaux militent activement auprès de milieux alternatifs, d'autres peuvent user de tactiques moins radicales qui peuvent néanmoins favoriser le changement social. Tel est le cas des travailleuses et travailleurs sociaux qui privilégient l'approche structurelle, et ce, même en milieux institutionnels. Malgré les obstacles pouvant se dresser sur le plan organisationnel, comme la lourdeur administrative et la surcharge de travail, ces derniers peuvent tout de même militer pour le changement social, tant à l'intérieur des murs de leur institution que dans la société (voir Mullaly, 2007, pour une discussion à cet égard).

Or, le militantisme doit actuellement être « revigoré » dans le domaine de l'intervention sociale afin d'en élargir la portée (Bent-Goodley, 2015). En effet, les intervenantes et intervenants peuvent s'en éloigner devant l'urgence des situations quotidiennes ou l'impuissance ressentie face à la complexité des problèmes sociaux. Dans ces circonstances, nous croyons nécessaire de contribuer à cette réflexion sur l'importance du militantisme dans le champ de l'intervention sociale et communautaire. Nous souhaitons ainsi que le présent numéro puisse continuer d'alimenter le dialogue sur l'apport du militantisme pour le changement social, notamment par des réflexions théoriques et pratiques de chercheures et chercheurs et d'intervenantes et intervenants dans le domaine du service social et de disciplines connexes.

Dans le premier numéro d'un volume de deux numéros portant sur le militantisme et le changement social, nous avons situé le militantisme et sa place dans le champ de l'intervention sociale et communautaire. Les différents textes constituant ce numéro se 
sont intéressés respectivement à la place du militantisme au sein de l'action collective et communautaire, aux pratiques liées à la défense des droits, au militantisme par l'entremise du témoignage public chez les communautés sexuelles et de genres, aux trajectoires d'engagement des femmes du secteur communautaire, à l'identification ethno-sexuelle chez les personnes LGBTQ+ d'origine libanaise, ainsi qu'à la participation des femmes à des comités de parents dans des maisons de naissance. Ce portrait varié et diversifié a permis de mettre en évidence le caractère indissociable du militantisme et de la pratique du service social, tout en offrant un aperçu de son opérationnalisation dans des contextes variés.

Le présent numéro permettra ainsi de poursuivre et d'approfondir la réflexion entamée dans le premier numéro du volume. Les textes qui le composent se penchent respectivement sur les personnes autistes en contexte linguistique minoritaire, sur le militantisme socio-sanitaire et le changement socio-spatial au Cameroun, sur la violence structurelle et les jeunes femmes de la rue, sur la marche "Les femmes, la rue, la nuit sans peur ", ainsi que sur la défense des droits des locataires. S'inscrivant en continuité du premier numéro, les différents articles réitèrent la pertinence et l'importance du militantisme dans le champ de l'intervention sociale et communautaire. Ils proposent tous, en trame de fond, des liens étroits entre l'intervention, le militantisme et le changement social.

Le numéro s'ouvre sur une entrevue avec Daniel Savoie, militant acadien qui a déployé différentes stratégies afin de favoriser l'accès des personnes à mobilité réduite au NouveauBrunswick et à l'Île-du-Prince-Édouard. De ses propos se dégagent une volonté de militer pour soi et pour les autres, mais également le désir de faire avancer la cause sur les plans social et politique. Dans cette entrevue, Savoie revient sur son parcours, ainsi que sur les principales batailles qu'il a menées au sein de sa profession et dans sa vie personnelle.

Cinq articles font partie du dossier, chacun d'entre eux abordant la question du militantisme et du changement social. Dans le premier article, Bianca Nugent rend compte d'une recherche portant sur les autistes en contexte linguistique minoritaire à Ottawa. Réalisé par l'entremise d'un blogue collaboratif, le discours des jeunes participants contraste avec les écrits scientifiques qui tendent à examiner les enjeux infantiles selon une approche développementale. Les implications pour le travail social et le militantisme sont abordées en conclusion.

Le deuxième article porte sur l'urbanisation, le militantisme socio-sanitaire et le changement socio-spatial au Cameroun. L'auteur, Dominique Meva'a Abomo, y présente les particularités du militantisme au sein de villes tropicales au Cameroun. Utilisant à 
la fois des données empiriques, des faits historiques et sa posture expérientielle comme chercheur, Meva'a Abomo retrace et aborde les obstacles liés à la santé communautaire du pays, ceux-ci étant le résultat complexe de la désarticulation structurelle et fonctionnelle du militantisme socio-sanitaire à l'intérieur du Cameroun.

Dans le troisième article, Catherine Flynn, Simon Lapierre, Pénélope Couturier et Marc Olivier Brousseau présentent les résultats d'une recherche-action participative réalisée avec de jeunes femmes de la rue, dans le but d'examiner les liens entre les violences structurelles et les violences vécues par ces femmes dans leurs relations intimes, en plus d'expérimenter une action pour lutter contre ces violences. L'article s'intéresse à la mobilisation de l'intersectionnalité des points de vue méthodologique et politique, qui se traduit notamment par une imbrication des savoirs universitaires et des savoirs expérientiels des participantes tout en soutenant la prise de parole de ces dernières.

Dans le quatrième article, Cécile Coderre et Sara-Maude Ivelyne Coderre brossent le portrait de la marche Take Back the Night (La rue, la nuit, femmes sans peur) depuis ses origines au milieu des années 1970. Dans un travail de recherche méticuleux dans plusieurs fonds d'archives, les auteures ont tenté de retracer les origines de cette marche en Europe et aux États-Unis, afin de mettre en lumière ses principales caractéristiques. Les auteures s'intéressent également à l'organisation de cette marche à Ottawa et à sa particularité : un comité francophone qui revendique l'amélioration des services en français en contexte minoritaire.

Dans le cinquième et dernier article, Geneviève Breault s'intéresse aux limites organisationnelles et aux pratiques démocratiques visant le développement des personnes mobilisées au sein de groupes communautaires montréalais de défense de droits des personnes locataires. Ayant réalisé sept entrevues avec des travailleuses et travailleurs qui œuvrent auprès d'organisations et de groupes qui défendent les droits des locataires, l'auteure révèle que la place accordée aux militantes et militants dans les espaces de délibération, ainsi que dans l'orientation des actions, alimente d'importants questionnements idéologiques et professionnels. Dans cet article, Breault propose un regard critique sur les modes d'appréhension de l'idéal démocratique dans un contexte de renouvellement des pratiques démocratiques.

Trois articles sur des pratiques novatrices composent la suite du numéro. Le premier article, rédigé par Geneviève L. Latour et C.Isabelle Gagnon, aborde la trajectoire qu’a suivie les organismes grassroots jeunesse et étudiants qui ont mené le dossier pour une université francoontarienne. Dans le deuxième article, Anne Jutras aborde les enjeux associés au militantisme en milieu francophone minoritaire. Plus particulièrement, elle 
présente un certain nombre de défis rencontrés par les intervenantes du Centre Novas - CALACS francophone de Prescott-Russell, ainsi que les stratégies développées pour sensibiliser la population au problème de la violence à caractère sexuel. Le troisième article est rédigé par Nérée St-Amand, qui revient sur son parcours professionnel, ainsi que sur les nombreuses réflexions qui l'ont habité tout au long de sa carrière. Son texte s'articule plus spécifiquement autour de la notion "d'aide ", qu'il met en lien avec le militantisme et le changement social, tout en soulignant des pratiques émergeant de réflexions politiques et sociales qu'il considère comme particulièrement inspirantes.

Bonne lecture!

\section{Bibliographie}

BENT-GOODLEY, Tricia B. (2015). «A Call for Social Work Activism », Social Work, Vol. 60, N 2, p. 101-103.

DESLAURIERS, Jean-Pierre, et Daniel TURCOTTE (2015). "Introduction », dans Jean-Pierre Deslauriers et Daniel Turcotte (dirs.), Introduction au travail social, Québec, Presses de l'Université Laval, p. 3-8.

FILLIEULE, Olivier, Éric AGRIKOLIANSKY et Isabelle SOMMIER (2010). Penser les mouvements sociaux : conflits sociaux et contestations dans les sociétés contemporaines, Paris, Éditions La Découverte, $327 \mathrm{p}$.

HANLEY, Jill, Anna KRUZYNSKI et Eric SHRAGGE (2013). « La place de «la communauté» dans le développement social : une politique néolibérale ou un site de transformation sociale? ", dans Elizabeth Harper et Henri Dorvil (dirs.), Le travail social : théories, méthodologies et pratiques, Québec, Presses de l'Université du Québec, p. 147-167.

LAPIERRE, Simon, et collab. (2017). " Le militantisme dans le champ de l'intervention sociale et communautaire ", Reflets: revue d'intervention sociale et communautaire, Vol. 23, № 1, p. 10-14.

LAVOIE, Jocelyne, et Jean PANET-RAYMOND (2014). La pratique de l'action communautaire, Québec, Presses de l'Université du Québec, 406 p.

MATHIEU, Lilian (2004). Comment lutter? Sociologie et mouvements sociaux, Paris, Éditions Textuel, $206 \mathrm{p}$.

MOLGAT, Marc (2015). «Définir, construire et redéfinir le travail social », dans Jean-Pierre Deslauriers et Daniel Turcotte (dirs.), Introduction au travailsocial, Québec, Presses de l'Université Laval, p. 9-28.

MULLALY, Bob (2007). The New Structural Social Work, Third Edition, Oxford University Press, 398 p.

RUSSELL, Bertie (2015). «Beyond Activism/academia: Militant Research and the Radical Climate and Climate Justice Movement(s) », Aera, Vol. 47, № 3, p. 131-148. 